\title{
Charge Transfer Excitation and Asymmetric Energy Transfer at the Interface of Pentacene-Perfluoropentacene Heterostacks
}

\author{
Anna-Katharina Hansmann, Robin C. Döring, Andre Rinn, Steffen M. Giesen, Melanie Fey, \\ Tobias Breuer, Robert Berger*, Gregor Witte*, and Sangam Chatterjee*
}

\begin{abstract}
High-performance solar cells demand efficient charge-carrier excitation, separation, and extraction. These requirements hold particularly true for molecular photovoltaics where large exciton binding energies render charge separation challenging at their commonly complex donor-acceptor interface structure. Amongst others, charge-transfer states are considered to be important precursors for exciton dissociation and charge-separation. However, the general nature of charge-transfer (CT) excitons and their formation pathways remain unclear. Layered quasi-planar crystalline molecular heterostructures of the prototypical donor-acceptor system pentacene-
\end{abstract}

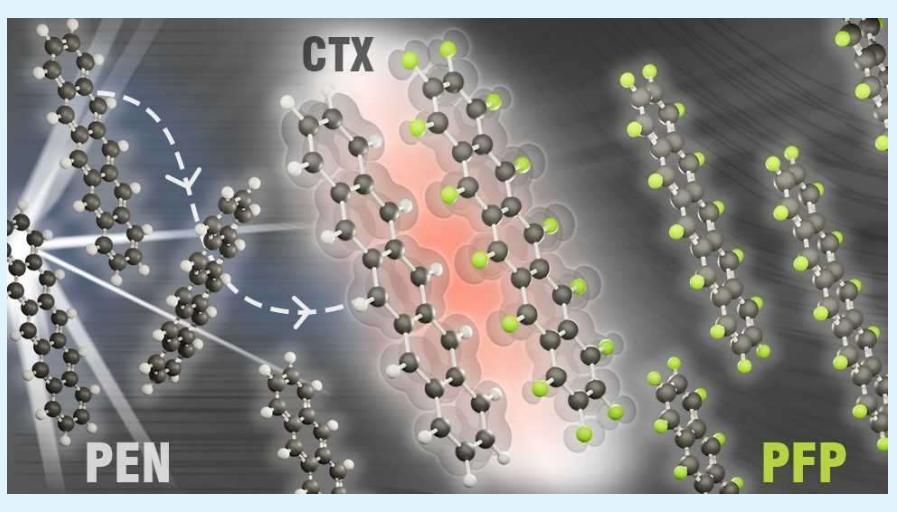
perfluoropentacene studied at cryogenic temperatures are a paramount model system to gain insight into the underlying physical mechanism. In particular, a detailed experiment-theory analysis showsthat exciton diffusion in unitary films influences the formation efficiency of CT excitons localized at internal interfaces for these conditions. The correlation of the structural characteristics, i.e., the molecular arrangement at the interfaces, with their absorption and photoluminescence excitation spectra is consistent with exciton transfer from pentacene to the charge-transfer exciton state only, whereas no transfer of excitons from the perfluoropentacene is detected. Electronic structure calculations of the model systems and investigation of coupling matrix elements between the various electronic states involvedsuggest hampered exciton diffusion towards the internal interface in the perfluoropentacene films. The asymmetric energy landscape around an idealized internal donor-acceptor interface thus is identified as a readon for asymmetric energy transfer. Thus, long-range effects apparently can influence charge separation in crystalline molecular heterostructures; similar to bandgap bowing which is well established for inorganic pn-junctions.

KEYWORDS: Organic semiconductor, molecular donor-acceptor pair, charge-transfer exciton, energy transfer, light matter in-teraction interaction

\section{- INTRODUCTION}

The microscopic characterization of organic $\mathrm{p}-\mathrm{n}$ heterojunctions based on molecular donor-acceptor pairs remains a challenging topic in current research despite their well-proven usage in novel device applications.(1-3) The versatility of synthetic chemistry provides numerous material systems ranging from small molecule donor-acceptors pairs to conjugated polymer blends. $(4,5)$ Many are targeted at organic photovoltaics (OPV) as perhaps one of the most important fields of application.(6,7) However, optical excitation of such molecular solids leads to the formation of charge-neutral excitons rather than the creation of free charge carriers due to the usually large exciton binding energies. As neutral particles cannot contribute to charge-current generation, this binding energy needs to be overcome for efficient current harvesting in photovoltaic devices. Charge-transfer (CT) excitons at the donor-acceptor interface, with the electron being transmitted to the acceptor moiety and the hole residing on the donor molecule, are prime candidates to help overcome this

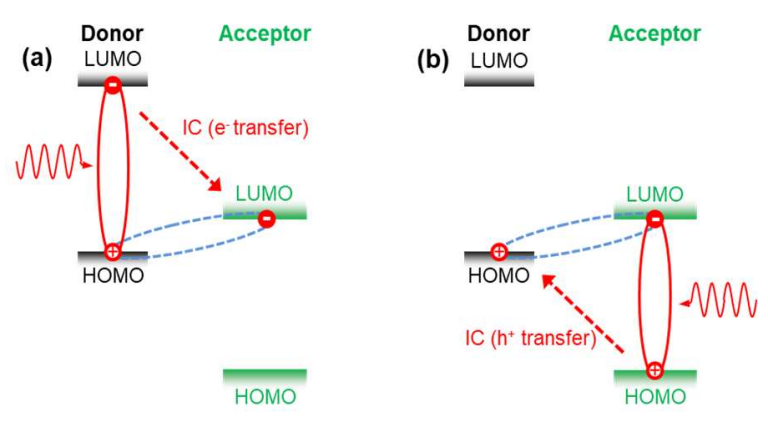

Figure 1 Schematic visualization of possible indirect excitation pathways for a CT exciton at the donor-acceptor interface according to the currently established CT exciton picture. Panel (a) displays a scheme of an optical excitation in the donor level, with subsequent relaxation of the electron in the (formerly) donor LUMO across the interface via internal conversion (IC). The case for an excitation in the acceptor layer is shown in panel (b). Here, the hole in the donor HOMO relaxes across the interface to form the charge-transfer exciton. 
energy barrier. While the process of CT exciton dissociation has been widely studied in many different ways, (2,7-12) the microscopic processes of CT exciton formation is less well investigated. Yet, understanding the mechanism leading to the population of chargetransfer exciton states is of crucial importance for attempts to enhance the performance of solar cells.(10,11,13-16) In a classical picture, the binding energy of these interfacial exciton states is frequently described by the energy difference between the highest occupied molecular orbital (HOMO) of the donor and lowest unoccupied molecular orbital (LUMO) of the acceptor molecule (sometimes referred to as hetero HOMO-LUMO),(14-16) with some corrections due to Coulomb interactions of both charge carriers, and the polarization of the crystal lattice. Sufficiently lowered exciton binding energies can be achieved by suitable alignment of the frontier orbitals of the constituting molecules at the interface. This should allow for efficient charge separation and, therefore, would enable current harvesting in OPV devices.(14-17) Thus, a high conversion rate from initial optical excitations to such CT-excitons appears to be desirable for high device efficiencies. However, the detailed nature of CT exciton states and their formation pathways remain generally unclear. In principle, such states can be populated either by electron transfer from the donor, by hole transfer from the acceptor or by a combination of both. Therefore, a thorough investigation of all states involved is necessary to draw more definite conclusions. The formation via electron-transfer after an initial excitation of the donor molecule (Figure 1a) is often discussed in the literature.(14-18) However, the hole-transfer from excited acceptor molecules (Figure 1b) is an equivalent pathway for the creation of CT-excitons, (12,19) especially when considering that many molecular solids discussed for applications in solar cells are p-type semiconductors.(19-21) Experimentally, it is challenging to determine which process prevails as CT-excitons commonly display characteristically low oscillator strength. This typically renders absorption-type optical measurements to be no viable option. In addition, lowenergy tails of much more intense higher-lying resonances, e.g., transitions attributed to exciton resonances of the respective unitary donor and acceptor materials my obscure the observation of CT excitons.

\section{- RESULTS AND DISCUSSION}

In this work, we investigate the formation pathways of CT-excitons at the model crystalline donor-acceptor interface of pentacene-perfluoropentacene (PEN-PFP) by a combination of advanced experimental and theoretical techniques. More specifically, we compare the one-photon photoluminescence excitation (PLE) and absorption spectra of three different PEN-PFP heterostructures with different molecular alignment at the interface at cryogenic temperatures. The emission (a)
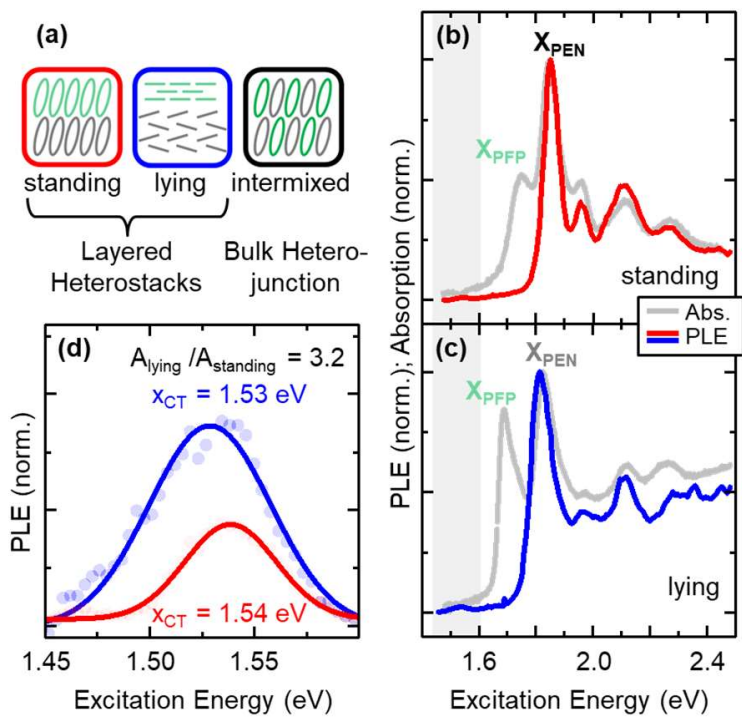

Figure 2 (a) Schematic depiction of the samples' arrangement of the three studied heterosystems. (b, c) Comparison of the absorption (grey) and PLE (color) spectra for the standing (red) heterostack and the lying (blue) heterostack at $20 \mathrm{~K}$ lattice temperature. The central detection wavelength for all measurements is $1.37 \mathrm{eV}(900 \mathrm{~nm}$,). (d) Detailed view of the PLE spectra of the two heterostack samples in the low-energy region (shaded area in panels $\mathrm{b}$ and $\mathrm{c}$ ). Lorentzian functions (solid lines) have been fitted to the experimental data (dots) in order to determine the respective peak areas A.

and exciton dynamics of these samples have been reported in our previous work.(22) The high sensitivity of PLE-spectroscopy allows us to examine both, the absorption energy of the CT-state itself as well as the coupling of all observed absorption features to the CTexciton system and, thereby, to obtain complementary information on its formation process. The PLE spectra in this work are obtained by measuring the photoluminescence intensity of the CT exciton depending on the photon energy of the exciting laser. The PLE signal intensity is given by:

$$
I_{\mathrm{PLE}}\left(E_{\mathrm{ex}}\right) \propto \alpha\left(E_{\mathrm{ex}}\right) \times \gamma \times P_{\mathrm{rad}} \times I_{\mathrm{ex}}\left(E_{\mathrm{ex}}\right),
$$

where $\alpha\left(E_{\varepsilon \xi}\right)$ is the absorption efficiency at the excitation energy $E_{\varepsilon \xi}, \gamma$ is the relaxation efficiency from the absorbing state to the emissive state, $P_{\text {rad }}$ is the radiative efficiency of the emitting state, and $I_{\varepsilon \xi}\left(E_{\varepsilon \xi}\right)$ is the excitation intensity. Generally, $P_{\text {rad }}$ is assumed to be independent of the excitation energy. Hence, any differences between the relative intensity of peaks observed in PLE and absorption are attributed to the coupling between the absorption and the CT-exciton state. (23)

Figure $2 \mathrm{a}$ displays structural schematics of the investigated molecular heterostructures. The molecules adopt either a standing or a lying molecular orientation at the internal interface. The former resembles an endon molecular arrangement of the interface molecules, 
whereas the latter displays a predominantly face-on alignment at the interface. (22) The structural details at the internal interface are as of yet unknown. Indications for a herringbone-type alignment in the lying heterostack are reported,(24) while the recent grazing incidence Xray diffraction study of a 1:1 blend of PEN: PFP finds a $\pi$-stack arrangement between PEN and PFP (25). The latter suggests that this motif can feature also in lying heterostacks while the former may potentially be the dominating motif directly at the interface or develops within the initial few layers of the bulk region.

For the sake of simplicity, we refer to these sample arrangements depicted in Figure $2 \mathrm{a}$ as standing heterostack and lying heterostack throughout the manuscript. In both cases, a PFP layer of $20 \mathrm{~nm}$ thickness has been grown on top of $20 \mathrm{~nm}$ of PEN. In addition, we also investigate an intermixed blend of both molecules in an equimolar ratio, in the following referred to as bulk heterojunction consistent with Ref. 22. Here, the molecules adopt an upright molecular orientation (total thickness $40 \mathrm{~nm}$ ) with respect to the substrate with a $\pi$-stack packing motif within the layers (25).

PL from the interfacial CT state of PEN-PFP heterostructures upon excitation with UV-light $(3.1 \mathrm{eV})$ has been previously reported at emission energies of $1.37 \mathrm{eV}$ and $1.35 \mathrm{eV}$ for standing and lying heterostacks, respectively.(22) Here, we take advantage of the high sensitivity of PLE measurements to determine the absorption energies related to the CT excitons. The corresponding PLE spectra are depicted in Figure $2 \mathrm{~b}$ and c. The detection window for the PLE spectra has been chosen to match the maximum of the CT emission from the heterostacks. Figure $2 \mathrm{~d}$ displays a detailed view of the PLE spectra near the expected CT transition. Indeed, a distinct resonance at around $1.54 \mathrm{eV}(1.53 \mathrm{eV})$ is observed in the PLE spectra of the standing (lying) heterostack. These values are in good agreement with the CT state energies reported for bulk heterostructures.(26-28) In contrast to the PLE spectra, the linear absorption spectra given in Figure $2 \mathrm{~b}$ and $\mathrm{c}$ (grey curves) show no indication of a CT state below the fundamental PFP resonance found at $1.75 \mathrm{eV}(1.69 \mathrm{eV})$ in the standing (lying) heterostack. The CT signals' intensity is assumingly below the sensitivity of our absorption setup.

Our accompanying theoretical description (see section Materials \& Methods for details of the different computational approaches) supports both, the assignment of the observed resonance to an interfacial CT state and its experimentally determined energy. Kohn-Sham orbital energies of PEN and PFP are strongly influenced by intermolecular interactions, which are most pronounced in the $\pi$-stacked molecular orientation. To mimic the situation at the interface theoretically, we arrange an increasing number of molecules in a $\pi$-stack, starting with a hetero dimer, up to an energy-optimized hexamer structure (see
Supporting Information for structural data). The energy of the CT state at the interface, when estimated based on Kohn-Sham orbital energies of the interfacial donoracceptor pair, seems to converge slowly as more and more molecules are added to the model. Via $1.55 \mathrm{eV}$ (hetero dimer) and $1.41 \mathrm{eV}$ (hetero tetramer), an estimated hetero HOMO-LUMO gap energy of $1.37 \mathrm{eV}$ is obtained in the hetero hexamer. This energy sequence of the different absorption signals is also well confirmed by computed energy expectation values of the excited states. For their computation we have used a nonorthogonal generalized group function (NOGF) ansatz, which is an ab-initio antisymmetrized direct product wavefunction approach $(29,30)$, where the excitation was localized to either the PEN or the PFP monomer, as described in the section Materials \& Methods. The resulting excitation energies with respect to the excitation localized at the PFP monomer are given as the diagonal matrix elements in Tables 1 to 3.

We compare quantitatively the experimental PLE signal strength related to the CT exciton for the different molecular orientations at the interface to facilitate a more detailed understanding of the electronic coupling situation at the internal interface. The ratio of the peak areas of the lying and standing heterostacks $A_{\text {lying }} / A_{\text {standing }}=3.2$ is in good agreement with the results from our previous work.(22) There, we find the intensity of the CT PL to be increased by a factor of three in the case of the lying heterostack. Hence, no additional recombination channels are present for the CT exciton, as its absorption and PLE signatures show similar intensity ratios. The increase of intensity in the lying arrangement is qualitatively confirmed by the transition probabilities resulting from the squared electric transition dipole moments $\mu^{2}$ of the CT-state calculated within time-dependent density functional theory (TDDFT). For this, an end-on heterodimer is used to mimic the structure at the interface in the standing heterostack, whereas two alternate arrangements, a $\square$-stacked and a herringbone heterodimer, are chosen as possible alignments representing the interface of the lying heterostack. The calculation yield ratios of $\left|\mu_{\pi \text {-stacked }}\right|^{2} /\left|\mu_{\text {end-on }}\right|^{2}=$ 15.2 and $\left|\mu_{\text {herringbone }}\right|^{2} /\left|\mu_{\text {end-on }}\right|^{2}=3.8$ using the energy-optimized heterodimer structures shown schematically in Figure 3.

$\left|\mu_{\pi \text {-stacked }}\right|^{2} /\left|\mu_{\text {herringbone }}\right|^{2}\left|\mu_{\text {head-to-tail }}\right|^{2}$ Next, $\quad$ we investigate the coupling of the different higher lying electronic states to the CT state. Therefore, we compare PLE spectra of the layered heterostacks with the respective absorption measurements. The absorption spectra in Figure 2b and c (grey curves) show pronounced features corresponding to the exciton resonances in the PEN and PFP layers, respectively. The exciton resonances are found at $1.75 \mathrm{eV}$ (PFP) and $1.85 \mathrm{eV}$ (PEN) for the standing heterostacks, while 
slightly shifted to lower energies of $1.69 \mathrm{eV}$ (PFP) and $1.82 \mathrm{eV}$ (PEN) in the lying heterostacks (cf. Ref. 22). Intriguingly, the PLE spectra for the CT emission taken in both heterostacks lacks a feature at energies associated with the lowest-energy PFP exciton resonance, whereas a resonance is clearly observed at energies associated with the lowest-energy absorption line in PEN. Consequently, the relaxation efficiency $\gamma$ from the absorbing state to the emissive CT state must be vanishingly small for excitation energies resonant to the lowest-energy PFP exciton transition. This apparently asymmetric energy transfer at the internal interface calls for an investigation of the underlying microscopic mechanisms as PLE data and $\gamma$ are just macroscopic observables.

Therefore, we test different hypotheses for the absence of the PFP exciton related features in the PLE spectra in the following: one option may be that the PFP-related excitations might be decoupled from the CT state due to a lack of the hole-transfer illustrated schematically in Figure 1b. While there could be an energetic argument in the standing heterostack, see below, this hypothesis appears unlikely as a general explanation. Our theoretical considerations provide no plausible argument, which could indicate the general disadvantage of an internal conversion related to hole transfer compared to electron transfer. PEN is an alternant hydrocarbon, and when PFP is considered as an inductively perturbed PEN in a simplified Hückel molecular orbital (HMO)-type of interpretation, their HOMO-LUMO gaps are expected to deviate only in second order HMO perturbation theory (cf. Ch. 2.4.1 in Ref. 31). The energy gap between donor HOMO and acceptor LUMO, in contrast, deviates much stronger and is also smaller than the HOMO-LUMO gap in the donor molecules and the gap between the frontier orbitals of the acceptor molecules. The formation of the CT state is thus feasible by energy considerations from both, the PEN exciton and the PFP exciton. The exception is the standing hetero stack, in which the charge separation over a large distance could energetically disfavor the CT state, as found for the model dimer structure (see Table 1 and description below).

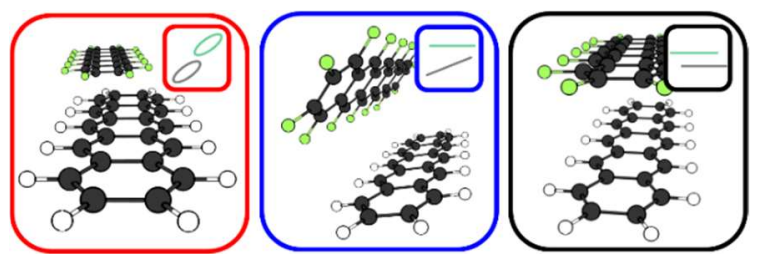

Figure 3: Molecular alignment of the dimers as obtained from structure energy-minimization and used in the computation of coupling matrices $\mathbf{V}$ given in Table 1 (red, end-on), 2 (blue, herringbone), and 3 (black, $\pi$-stacked), together with schematic representations in insets.
Another explanation for the observed asymmetry could be a significantly different electronic coupling situation. To test this hypothesis theoretically, the matrix $\boldsymbol{V}$ is calculated in the NOGF approach described in the Computational Methods. $\boldsymbol{V}$ is given in Tables 1 to 3 for the three intermolecular orientations shown in Figure 3. The off-diagonal elements $V_{i j}(i \neq j)$ are coupling matrix elements, quantities related in a generalized way to the well-known charge-transfer integrals, and their squares are proportional to the transition rate (cf., e.g., Ref. 32). Here, we calculate $V_{i j}$ for transitions between initial excitation configurations localized in the respective acceptor and donor molecules, $\left|\Phi_{\text {Pen }}^{(0)} \Phi_{\text {PFP }}^{(*)}\right\rangle$ and $\left|\Phi_{\text {Pen }}^{(*)} \Phi_{\text {PFP }}^{(0)}\right\rangle$, as well as for the final CT configuration $\left|\Phi_{\text {Pen }}^{(+)} \Phi_{\text {PFP }}^{(-)}\right\rangle$. The diagonal elements $V_{i i}$ are the energy expectation values of these configurations. Coupling of the two former localized states to the CT configuration is, although changing significantly in magnitude for different molecular orientations, always of similar order of magnitude for the two excitation localized in PEN or PFP. Hence, there is no indication for decoupling of PFP excitations from the CT exciton system for the dimer structures employed herein. It is known, however, that the transfer integrals depend on the specific molecular arrangement (33). Since the structure of the co-crystal of the blend of PEN and PFP became recently available, we also calculated the coupling for the $\pi$ stacked dimer taken from the crystal structure, which also provide no support for the discussed decoupling.

Table 1. Matrix $\boldsymbol{V}$ containing the calculated energy expectation values and electronic coupling matrix elements of the end-on molecular orientation. All energies are given in $\mathrm{eV}$ and in reference to one of the excited configurations.

\begin{tabular}{cccc}
\hline 0 & $\left|\Phi_{\mathrm{Pen}}^{(0)} \Phi_{\mathrm{PFP}}^{(*)}\right\rangle$ & $\left|\Phi_{\mathrm{Pen}}^{(*)} \Phi_{\mathrm{PFP}}^{(0)}\right\rangle$ & $\left|\Phi_{\mathrm{Pen}}^{(+)} \Phi_{\mathrm{PFP}}^{(-)}\right\rangle$ \\
\hline$\left\langle\Phi_{\mathrm{Pen}}^{(0)} \Phi_{\mathrm{PFP}}^{(*)}\right|$ & 0 & $-2.2 \times 10^{-3}$ & $1.7 \times 10^{-4}$ \\
\hline$\left\langle\Phi_{\mathrm{Pen}}^{(*)} \Phi_{\mathrm{PFP}}^{(0)}\right|$ & $\begin{array}{l}-2.2 \\
\end{array}$ & $1.1 \times 10^{-1}$ & $-1.6 \times 10^{-4}$ \\
\hline$\left\langle\Phi_{\mathrm{Pen}}^{(+)} \Phi_{\mathrm{PFP}}^{(-)}\right|$ & $1.7 \times 10^{-4}-1.6 \times 10^{-4}$ & $4.5 \times 10^{-2}$ \\
\hline
\end{tabular}

Table 2. Matrix $\boldsymbol{V}$ containing the calculated energy expectation values and electronic coupling matrix elements of the herringbone molecular orientation. All energies are given in $\mathrm{eV}$ and in reference to one of the excited configurations.

\begin{tabular}{cccc}
\hline$\square$ & $\left|\Phi_{\text {Pen }}^{(0)} \Phi_{\text {PFP }}^{(*)}\right\rangle$ & $\left|\Phi_{\text {Pen }}^{(*)} \Phi_{\text {PFP }}^{(0)}\right\rangle$ & $\left|\Phi_{\text {Pen }}^{(+)} \Phi_{\text {PFP }}^{(-)}\right\rangle$ \\
\hline$\left\langle\Phi_{\text {Pen }}^{(0)} \Phi_{\text {PFP }}^{(*)}\right.$ & 0 & $6.8 \times 10^{-2}$ & $1.4 \times 10^{-2}$ \\
\hline$\left\langle\Phi_{\text {Pen }}^{(*)} \Phi_{\text {PFP }}^{(0)}\right|$ & $6.8 \times 10^{-2}$ & $1.1 \times 10^{-1}$ & $1.4 \times 10^{-2}$ \\
\hline$\left\langle\Phi_{\text {Pen }}^{(+)} \Phi_{\text {PFP }}^{(-)}\right|$ & $1.4 \times 10^{-2}$ & $1.4 \times 10^{-2}$ & $-7.1 \times 10^{-1}$ \\
\hline
\end{tabular}

Table 3. Matrix $\boldsymbol{V}$ containing the calculated energy expectation values and electronic coupling matrix elements of the $\pi$ stacked molecular orientation. All energies are given in $\mathrm{eV}$ and in reference to one of the excited configurations. 


\begin{tabular}{cccc}
\hline$\square$ & $\left|\Phi_{\mathrm{Pen}}^{(0)} \Phi_{\mathrm{PFP}}^{(*)}\right\rangle$ & $\left|\Phi_{\mathrm{Pen}}^{(*)} \Phi_{\mathrm{PFP}}^{(0)}\right\rangle$ & $\left|\Phi_{\mathrm{Pen}}^{(+)} \Phi_{\mathrm{PFP}}^{(-)}\right\rangle$ \\
\hline$\left\langle\Phi_{\mathrm{Pen}}^{(0)} \Phi_{\mathrm{PFF}}^{(*)}\right.$ & 0 & $8.8 \times 10^{-2}$ & $4.8 \times 10^{-2}$ \\
\hline$\left\langle\Phi_{\mathrm{Pen}}^{(*)} \Phi_{\mathrm{PFF}}^{(0)}\right.$ & $8.8 \times 10^{-2}$ & $1.0 \times 10^{-1}-7.6 \times 10^{-3}$ \\
\hline$\left\langle\Phi_{\mathrm{Pen}}^{(+)} \Phi_{\mathrm{PFP}}^{(-)}\right|$ & $4.8 \times 10^{-2}$ & $-7.6 \times 10^{-3}-7.4 \times 10^{-1}$ \\
\hline
\end{tabular}

As third hypothesis, we thus consider the electronic situation at and around the interface, which is described by the excitation energies of the lowest PEN and PFP excitons in our hexamer model for the layered heterostack. Here, we introduce two models of varying complexity; both find significant differences in the transfer of excitons towards the interface between PEN and PFP. The first one is straight forwardly based on the molecular orbital energies of three PEN and three PFP molecules being in a $\pi$-stacked arrangement. Figure $4 \mathrm{a}$ schematically depicts the resulting HOMO-LUMO gaps. They suggest a downhill slope of the energies associated with the exciton state away from the interface towards the unitary films for PFP, whereas the corresponding slope nearly vanishes or is slightly downhill towards the interface on the PEN side. This effect appears similar to band-bending.(34)

The corresponding energy gradient on the PFP side acts as an activation barrier ( $E_{\text {Barrier }}$ ) for exciton diffusion towards the interface region. At cryogenic temperatures, the thermal energy is expected to be less than the energy barrier for PFP, effectively confining PFP excitons to the bulk of the PFP layer. Consequently, only those PFP excitons created directly at the interface are able to relax to the CT state. In the case of PEN, the lacking energy barrier would allow the excitons created in the bulk to diffuse to the interface and, thus, populate the CT state. As the vast majority of excitons are created in the unitary films of the sample, and not directly at the interface, the PFP excitons cannot contribute significantly to the measured CT PL signal.

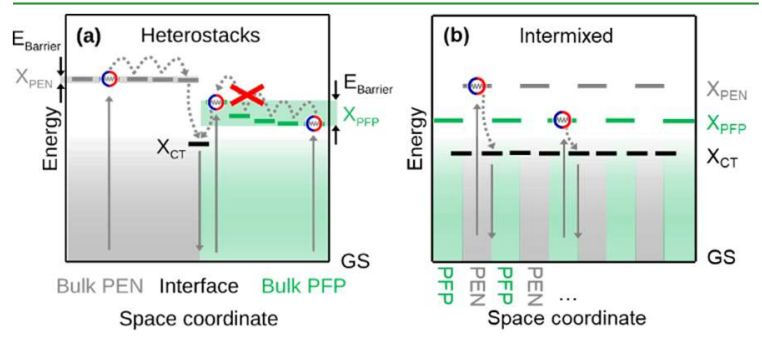

Figure 4 Schematic depiction of the energy level alignments for the lowest energy exciton states at the PEN-PFP interface, in the layered heterostacks (a) and the intermixed bulk heterojunctions (b).

A more elaborate theoretical description of the electronic structure shows that the energy barrier for the PEN layer may even vanish: we calculate diabatic excitation energies using the same approach of localized states as for the coupling matrices. Unitary films and interface regions are simulated by extending the dimer antisymmetrized product wave functions of Eq. (2) to a one dimensional $\pi$-stacked hexamer model $\left|\Phi_{\mathrm{Pen}_{1}}^{m_{1}} \Phi_{\mathrm{Pen}_{2}}^{m_{2}} \Phi_{\mathrm{Pen}_{3}}^{m_{3}} \Phi_{\mathrm{PFP}_{4}}^{m_{4}} \Phi_{\mathrm{PFP}_{5}}^{m_{5}} \Phi_{\mathrm{PFP}_{6}}^{m_{6}}\right\rangle$ as prescribed by Eq. (3) (see Materials \& Methods). Here, the monomer at index $i$ is in the electronic state $m_{i}$ with $1 \leq i \leq 6$. To model the movement of the exciton from the unitary layer to the interface, the states are prepared such that the excitation is localized on the $k$-th monomer. Then, $m_{k}$ is the first excited singlet state and $m_{i}$ the electronic ground state for all $i \neq k$. These results also indicate an activation barrier for PFP, which is of similar magnitude as for HOMO-LUMO energy differences schematically shown in Figure 4b. For PEN, however, this model predicts a slight decrease in energy for the excitation localized at the PEN monomer positioned directly at the interface. This indicates a transfer of excitation from the unitary PEN film to the interface without activation barrier (see Supporting Information for details on the results of both theoretical approaches and the results for a further extended dodecamer model system). Thus, our results suggest that different diffusion properties of the excitons from the two unitary film regions to the interface could serve as an explanation for the asymmetric energy transfer, evidenced by the absence of the PFP exciton resonance in the PLE spectra.

To provide further evidence for hampered diffusion, we now turn to the bulk heterojunction. As shown previously, both molecules adopt a perfect intermixture with a cofacial stacking of PEN and PFP molecules in such equimolar blends (25) ( $c f$. Figure 2a). In the present case, the molecules adopt an upright molecular orientation (total thickness $40 \mathrm{~nm}$ ) with respect to the quartz substrate. The hypothesis of hampered diffusion is reinforced by investigation of such bulk heterojunctions: a perfectly intermixed sample resembles the case of infinite interfaces, as the unit cell should consist of equal number of molecule of each type. Consequently, the population of the CT state should be independent of diffusion properties, as both PEN and PFP excitons can reach the molecular heterointerface without prior diffusion, as illustrated schematically in Figure 4b. Figure $5 \mathrm{a}$ gives the lowtemperature PL spectrum of the bulk heterojunction along with the PL spectra of unitary samples of PEN and PFP, respectively. All data have been obtained under rigorously comparable experimental conditions. The spectrum of the bulk heterojunction sample shows no emission related to the unitary phases of PEN and PFP. Only the CT PL at $1.4 \mathrm{eV}$ and a shoulder at $1.55 \mathrm{eV}$ is observed, much lower in energy than the main emission lines of the so-called free exciton (FX) at $1.82 \mathrm{eV}$, the emission frequently ascribed to a self-trapped exciton (STX) at $1.65 \mathrm{eV}$ of the standing PEN film, and the corresponding PFP free exciton at $1.71 \mathrm{eV}$. (22) This is consistent with the formation of a new crystal structure and the formation of the associated electronic band 
structure. (25) Regardless, in a simplistic view, emission related to unitary PFP should be observable in the PL of the bulk heterojunction if PFP excitations were decoupled from the CT state; however, this emission is not observed in our experiments. It should be noted, however, that the signal attributed to unitary PFP also vanishes in the standing heterostack (22), which would be consistent with the calculated increase in CT state energy for the model dimer system with end-on arrangement (see Table 1).

Additionally, the effect would also be observable when comparing the PLE spectra and the absorption measurements of the intermixed bulk heterojuntion; Figure $5 \mathrm{~b}$ provides the respective data. Although the individual resonances have not been assigned to the different electronic states with full certainty to date, the data still provides valuable information: all peaks observed in the absorption spectrum are reproduced in the PLE spectrum, confirming efficient coupling to the CT state. This is true for all resonances in the intermixed sample, regardless whether they might be related to PEN or PFP. Only at higher excitation energies, above $2.1 \mathrm{eV}$, the PLE signal decreases compared to the absorption data. This indicates that the competition between radiative and non-radiative decay strongly depends on the excess excitation energy deposited to the system. Regardless of these aspects, our findings indicate efficient coupling of PFP excitons to the CT state, which reinforces the hypothesis of hampered diffusion in the layered heterostacks.

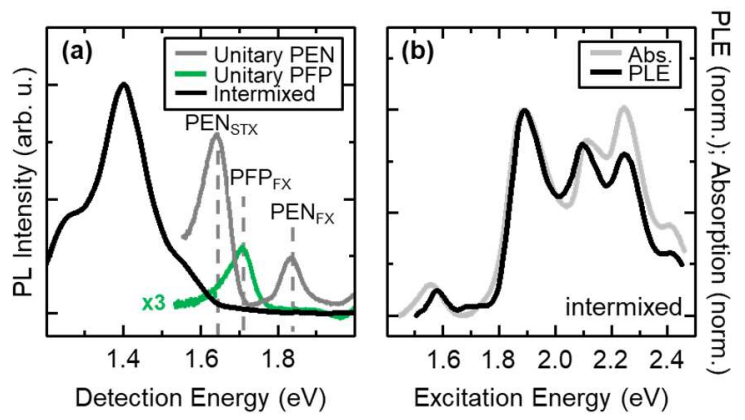

Figure 5. (a) PL spectrum of the intermixed sample (black) recorded at $4 \mathrm{~K}$ lattice temperature and excited at $3.1 \mathrm{eV}$. For reference, spectra of uprightly orientated unitary PEN (grey) and PFP (green) films of $20 \mathrm{~nm}$ thickness obtained under the same experimental conditions are also given. The PL spectra are taken from our previous study.(22) (b) Comparison of the absorption (grey) and PLE (black) spectra for the intermixed bulk heterojunction of uprightly oriented molecules with a total thickness of $40 \mathrm{~nm}$.

\section{- CONCLUSIONS}

In conclusion, our work shows an intriguing asymmetric energy transfer in different model PEN-PFP heterostacks. Decoupling of excitons in PFP films and the CT exciton at the interface is excluded as origin of the asymmetry by means of different quantum chemical calculations and detailed spectroscopic investigations of the bulk heterojunctions. Instead, the hypothesis of hampered diffusion of excitons in unitary PFP films to the internal interface region suggested by different theoretical models supports the various experimental spectroscopic observations in heterostacks as well as control experiments on intermixed bulk heterojunctions. These findings underline the crucial role played by internal interfaces between molecular semiconductor materials and the particular need for a thorough understanding of the underlying interfacial electronic coupling situations in molecular heterojunctions. The underlying physical mechanisms behind such effects are not immediately obvious, neither from an energy nor from a coupling-based perspective alone. Hence, they provide an example of the challenges involved in the study of molecular $\mathrm{p}-\mathrm{n}$ heterojunctions, and how they can be overcome. This highlights the importance of a multifaceted combined experimental and theoretical approach under idealized conditions such as idealized, crystalline structures studied under cryogenic conditions structures to reveal the electronic structure and excitation dynamics at organic interfaces in general.

\section{- EXPERIMENTAL SECTION}

Sample Preparation. The PEN (Sigma-Aldrich) and PFP (Kanto Denka Kogyo Co.) films are grown under high-vacuum conditions by molecular-beam deposition from resistively heated Knudsen cells. The molecular flux during film growth is monitored by a quartz crystal microbalance and typically balanced at $6 \AA / \mathrm{min}$. Unitary films with upright molecular orientation are prepared on quartz substrates. Here, the crystalline PEN films adopt the thin-film phase, while PFP crystallizes in the bulk polymorph. $(35,36)$ In contrast, acene films reveal a lying molecular orientation on graphene-covered quartz $(21,37)$ like in the case of graphite substrates.(38) In this case, PFP crystallizes in a $\square$-stacked polymorph where all molecules are slip-stacked with their molecular plane parallel to the substrate surface.(21) PEN crystallizes in the Siegrist phase (39) on graphene and reveals $(0 \overline{2} \overline{2})$ and $(1 \overline{2} \overline{1})$ oriented films where the long axis of the molecules are oriented essentially parallel to the substrate surface.(38) Mixed bulk heterostructures (blends) are prepared by simultaneous deposition ("codeposition") of PEN and PFP with equimolar stoichiometry onto a quartz substrate yielding well intermixed blends where all molecules reveal an exclusive upright orientation. Heterostacks of upright and lying molecular orientation with high degrees of uniformity and crystallinity are achieved by first growing uniformly oriented PFP films on the various substrates, which are then used as template for the subsequent growth of PEN layers. As demonstrated in previous work, this concept of inheriting molecular orientation allows the fabrication of heterostacks with uniform molecular orientation and well-defined interfaces.(40) The films are prepared with nominal thicknesses of $20 \mathrm{~nm}$ for the unitary films and $40 \mathrm{~nm}$ for the heterostructures (in each case with stoichiometric mixture of both constituents). The substrates are kept at room temperature during film preparation to 
minimize molecular intermixture at the interface for the layered heterostructures.(28) All film structures are verified by $\mathrm{X}$-ray diffraction analyses.

Spectroscopic methods. A Ti:sapphire laser oscillator emitting 30-fs pulses at a repetition rate of $80 \mathrm{MHz}$ at a wavelength of $800 \mathrm{~nm}$ is used to pump a photonic crystal fiber (PCF). The PCF generates a white-light supercontinuum covering the entire visible and near-infrared spectral regions with a total output power of $150 \mathrm{~mW}$. Continuously tunable excitation wavelengths are achieved by dispersing the supercontinuum with a home-built prism monochromator yielding a spectral resolution of $1 \mathrm{~nm}$. The monochromatic excitation light is focused to a spot size of $150 \mu \mathrm{m}$. The sample is mounted in vacuum in a helium closed cycle cryostat and is cooled to $20 \mathrm{~K}$. The emitted PL light is collected by a large numerical aperture optic. A grating spectrograph $(320 \mathrm{~mm}$ focal length) is used for dispersion of the PL and detection is performed with a charge-coupled device cooled to $200 \mathrm{~K}$. A small portion $(\sim 4 \%)$ of the excitation light is guided towards a calibrated Si-photodiode and excitation power is monitored throughout the data acquisition process. Collected data are corrected for excitation power and spectral response of the detection scheme. From the obtained 2D data transients from different detection wavelength regions can be extracted and yield the presented PLE spectra. Absorption measurements have been performed in transmission geometry using the PLE setup, replacing the exciting laser with a tungsten halogen lamp. The light is focused on the sample to a spot size of about $150 \mu \mathrm{m}$. The measured transmission spectra have been referenced against a part of the substrate of each individual sample with no PEN-PFP coverage. The absorption is given by $A=1-T=1-\left(I_{\text {sample }}-I_{\text {background }}\right) /\left(I_{\text {reference }}-I_{\text {background }}\right)$.

Computational Methods. To obtain molecular structures and orbital energies, the monomeric building blocks PEN and PFP as well as hetero dimers, tetramers and hexamers as models for the layered heterostuctures and intermixed bulk heterojunction are investigated with the program Turbomole 7.0 (41) on the level of DFT (B3-LYP) $(29,42-45)$ using the def2-TZVP basis set.(46) Numerical integrations are performed on an $\mathrm{m} 4$ level grid for the dimers, whereas the $\mathrm{m} 3$ level grid is selected for the tetramer and hexamer structures. The self-consistent field convergence criterion is chosen to be $10^{-6} E_{\mathrm{h}}$. The structures have been energy-minimized up to a gradient norm below the convergence criterion of $10^{-4} \mathrm{E}_{\mathrm{h}} / \mathrm{a}_{0}$ for all systems besides the hexamer, for which this criterion has been relaxed to $10^{-3} \mathrm{E}_{\mathrm{h}} / \mathrm{a}_{0}$. TDDFT calculations are performed with the program ORCA(47) using the long-range corrected hybrid functional CAM-B3LYP(48) and the def2-TZVP basis set.(46)

Various dimer arrangements are energy-minimized on the DFT level. Assuming a similar orientation as in PEN and PFP bulk structures, $\pi$-stacked, herringbone and head-to-tail arrangements are chosen for further investigation of coupling matrix elements, even though it is known that the structure of the lying heterostack should probably be described as an intermediate between the well-known $\pi$-stacked and herringbone motif.(40) All structures exceeding the dimer model were composed in $\pi$-stacks, as this arrangement has largest intermolecular overlap between electronic wave functions of the building blocks and therefore the strongest coupling effects.

Beyond the simple molecular Kohn-Sham orbital model, we use an ab-initio wave function approach for the calculation of the coupling matrix elements presented in Table 1 to 3 . Antisymmetrized product wave functions $\left|\Phi_{\kappa}\right\rangle$ are constructed that consist of independent monomer wave functions $\left|\Phi_{\mathrm{M}}^{m}\right\rangle$, of the systems $M=A, B, C, \ldots$ in localized states $m=$ $a, b, c, \ldots$ with $\kappa=A a, B b, C c, \ldots$ denoting the selection of systems and states. $(49,50)$ Since monomer wave functions are in this scheme not influenced by each other, this approach can be used to build up systematically the bulk and interface regions. Our specific concept and implementation will be described in detail elsewhere. To outline briefly some of the general ideas: The interface is modeled as a dimer to compute the coupling matrix elements and as a one dimensional $\pi$ stacked hexamer to determine diabatic excitation energies. The states contributing to the coupling matrix are selected to $\mathrm{m} \#$ odel the interface characteristics. The resulting dimer wave function for the product of two systems A and B in states $a$ and $b$ is obtained as

$\left|\Phi_{A a B b}\right\rangle=\left|\Phi_{A}^{a} \Phi_{B}^{a}\right\rangle=N_{A a B b} \hat{A}\left[\left|\Phi_{A}^{a}\right\rangle\left|\Phi_{B}^{b}\right\rangle\right]$,

with normalization factor $N_{\mathrm{A} a \mathrm{~B} b}$ and antisymmetrization operator $\hat{A}$.

For a general interface model of two systems $R$ and $S$, composed of $N_{R}$ and $N_{S}$ monomers $R_{i}$ and $S_{i}$, respectively, in states $m_{i}$. the wave function is

$\left|\Phi_{\mathrm{K}}^{R S}\right\rangle=N_{\mathrm{K}} \hat{A}\left|\prod_{i}^{N_{R}} \Phi_{R_{i}}^{m_{i}} \prod_{i=N_{R}+1}^{N=N_{R}+N_{S}} \Phi_{S_{i}}^{m_{i}}\right\rangle$.

To examine CT at the PEN-PFP interface, the electronic configurations of the monomers employed are chosen to be the Hartree-Fock ground state configurations for PEN $\left|\Phi_{\text {Pen }}^{(0)}\right\rangle$ and for PFP $\left|\Phi_{\text {PFP }}^{(0)}\right\rangle$, the HOMO-LUMO singlet excitation configuration state function (CSF) localized at PEN $\left|\Phi_{\text {PEN }}^{(*)}\right\rangle$ and at PFP $\left|\Phi_{\text {PFP }}^{(*)}\right\rangle$ and the localized PEN cation and PFP anion configurations, $\left|\Phi_{\text {Pen }}^{(+)}\right\rangle$and $\left|\Phi_{\text {PFP }}^{(-)}\right\rangle$respectively. The normalized and antisymmetrized products of these configurations, $\left|\Phi_{\mathrm{Pen}}^{(0)} \Phi_{\mathrm{PFP}}^{(*)}\right\rangle,\left|\Phi_{\mathrm{Pen}}^{(*)} \Phi_{\mathrm{PFP}}^{(0)}\right\rangle$ and $\left|\Phi_{\mathrm{Pen}}^{(+)} \Phi_{\mathrm{PFP}}^{(-)}\right\rangle$, are then used as approximations to the investigated states, i.e. the excitons at the interface, localized at PEN and at PFP, and the CT state, respectively. The coupling matrix elements are obtained from the off-diagonal elements of the resulting configuration interaction matrix $\boldsymbol{H}$, with elements $H_{\kappa \lambda}=$ $\left\langle\Phi_{\kappa}|H| \Phi_{\lambda}\right\rangle$, where $H$ is in the present application restricted to the non-relativistic electrostatic Hamiltonian.

This approach allows us to use a flexible multi-electron wave function basis for the description of the various electronic states involved. This means that the monomer wave functions can in principle be described at any required level of complexity, including even a full configuration interaction treatment. Herein, however, the monomer wave functions are approximated by determinants optimized on the restricted open-shell Hartree-Fock (ROHF) level. As the singly-excited singlet configurations $\left|\Phi_{\mathrm{M}}^{(*)}\right\rangle$ are not Roothaan cases, the molecular orbitals are optimized for the corresponding ROHF triplet case instead and subsequently used to form the singlet CSFs. Consequently, all monomer wave functions are spinadapted to yield the required singlet configurations of the dimer by using linear combinations of determinants.

Since the monomer wave functions here result from independent calculations with different and not necessarily orthogonal sets of orbitals, relaxation effects are included. The calculation of $H_{\kappa \lambda}$ with these non-orthogonal product wave functions then makes it necessary to apply the so-called 
Löwdin rules for non-orthogonal orbitals.(51) Subsequently, $\boldsymbol{H}$ is symmetrically orthogonalized as $\forall=S^{-1 / 2} H S^{-1 / 2}$, where $\boldsymbol{S}$ is the configuration overlap matrix with elements $S_{\kappa \lambda}=\frac{\left\langle\Phi_{\kappa} \mid \Phi_{\lambda}\right\rangle}{\left(\left\langle\Phi_{\kappa} \mid \Phi_{\kappa}\right\rangle\left\langle\Phi_{\lambda} \mid \Phi_{\lambda}\right\rangle\right)^{1 / 2}}$. The orthogonalization procedure can induce a change in the corersponding elements of $V_{\text {when }}$ adding or removing configurations in the selection, which will be small as long as the configurations are already nearly orthogonal. Finally, $V=V-V_{11} 1_{3 \times 3}$ (where 1 is the identity matrix) is the matrix reported in Tables 1 to 3 .

For all monomer wave function optimizations, the positions of the nuclei have been kept fixed to the dimer structure obtained as described above (and given in the Supporting Information). The ROHF wave functions of the monomers are then calculated independently, using Turbomole (40) using the def2-SVP basis set. (52) The method for the calculation of coupling matrix elements of the antisymmetrized product wave functions of these monomer wave functions has been implemented into the program nonorth, a tool box for calculations employing multiple, nonorthogonal wave functions.

\section{- ASSOCIATED CONTENT}

\section{Supporting Information}

The supporting information contains absorption spectra of the unitary films. Additionally, schematic representation of orbital energies and excitation energies of the heterohexamer model are provided. Cartesian coordinates of the various structures used in this study are provided. This material is available free of charge via the Internet at http://pubs.acs.org.

\section{- AUTHOR INFORMATION Corresponding Authors \\ Sangam Chatterjee - Institute of Experimental Physics I and Center for Materials Research (LaMa), Justus Liebig University Giessen, Heinrich-Buff-Ring 16, D- 35392 Giessen, Germany \\ Email: *sangam.chatterjee@physik.uni-giessen.de \\ Gregor Witte - Department of Physics and Materials Sciences Center, Philipps-Universität Marburg, Renthof 7, D-35032 Marburg, Germany \\ Email: *gregor.witte@physik.uni-marburg.de \\ Robert Berger - Department of Chemistry, Philipps- \\ Universität Marburg, Hans-Meerwein-Straße 4, \\ D-35032 Marburg, Germany \\ Email: robert.berger@chemie.uni-marburg.de}

\section{Authors}

Anna-Katharina Hansmann - Department of Chemistry, Philipps-Universität Marburg, Hans-MeerweinStraße 4, D-35032 Marburg, Germany

Robin C. Döring - Institute of Experimental Physics I and Center for Materials Research (LaMa), Justus Liebig University Giessen, Heinrich-Buff-Ring 16, D35392 Giessen, Germany

Andre Rinn - Institute of Experimental Physics I and Center for Materials Research (LaMa), Justus Liebig University Giessen, Heinrich-Buff-Ring 16, D-35392 Giessen, Germany

Steffen M. Giesen - Department of Chemistry,
Philipps-Universität Marburg, Hans-Meerwein-

Straße 4, D-35032 Marburg, Germany

Melanie Fey - Institute of Experimental Physics I and

Center for Materials Research (LaMa), Justus Liebig

University Giessen, Heinrich-Buff-Ring 16, D-35392

Giessen, Germany

Tobias Breuer - Department of Physics and Materials Sciences Center, Philipps-Universität Marburg,

Renthof 7, D-35032 Marburg, Germany

Complete contact information is available at:

\section{Author Contributions}

TB and GW prepared the samples and provided the structural characterization. RD, AR, and SC performed all spectroscopic experiments and analyzed the data. AKH, SMG, and RB provided the theoretical results and helped interpret the experimental data. The manuscript has been written through contributions of all authors. All authors have given approval to the final version of the manuscript.

\section{Notes}

The authors declare no competing financial interest.

\section{- ACKNOWLEDGEMENTS}

Financial support was provided by the German Science Foundation (Deutsche Forschungsgemeinschaft, DFG) project-ID 223848855-SFB 1083 "structure and dynamics of internal interfaces" within projects A2, B2 and B8. SC also acknowledges the Heisenberg Programme (CH660/8)

\section{- REFERENCES}

(1) Sakamoto, Y.; Suzuki, T.; Kobayashi, M.; Gao, Y.; Fukai, Y.; Inoue, Y.; Fumio Sato; Tokito, S. Perfluoropentacene: High-Performance P-N Junctions and Complementary Circuits with Pentacene. J. Am. Chem. Soc. 2004, 126, 81388140.

(2) Jailaubekov, A. E.; Willard, A. P.; Tritsch, J. R.; Chan, W.; Sai, N.; Gearba, R.; Kaake, L. G.; Williams, K. J.; Leung, K.; Rossky, P. J.; et al. Organic Photovoltaics. Nature Mater. 2012, 12, 66-73.

(3) Park, K. S.; Lee, K. S.; Kang, C. M.; Baek, J.; Han, K. S.; Lee, C.; Koo Lee, Y. E.; Kang, Y.; Sung, M. M. Cross-Stacked Single-Crystal Organic Nanowire P-N Nanojunction Arrays by Nanotransfer Printing. Nano Lett. 2015, 15, 289-293.

(4) Congreve, D. N.; Lee, J.; Thompson, N. J.; Hontz, E.; Yost, S. R.; Reusswig, P. D.; Bahlke, M. E.; Reineke, S.; Van Voorhis, T.; Baldo, M. A. External Quantum Efficiency Above $100 \%$ in a Singlet-Exciton-Fission-Based Organic Photovoltaic Cell. Science 2013, 340, 334-337.

(5) Facchetti, A. pi-Conjugated Polymers of Organic Electronics and Photovoltaic Cell Applications. Chem. Mater. 2011, 23, 733-758

(6) Inganäs, O. Organic Photovoltaics over Three Decades. Avd. Mater. 2018, 30, 1800388 and references therein.

(7) Hedley, G. J.; Ruseckas, A.; Samuel, I. D. W. Light Harvesting for Organic Photovoltaics. Chem. Rev. 2017, 117, 796-837.

(8) Stoltzfus, D. M.; Donaghey, J. E.; Armin, A.; Shaw, P.E.; Burn, P. L.; Meredith, P.; Charge Generation Pathways in 
Organic Solar Cells: Assessing the Contribution from the Electron Acceptor, Chem. Rev. 2016, 116, 12920-12955

(9) Street, R. A.; Electronic Structure and Properties of Organic Bulk-Heterojunction Interfaces, Adv. Mater. 2016, 28, 3814-3830.

(10) Few, S.; Frost, J. M.; Nelson, J.; Models of charge pair generation in organic solar cells, Phys. Chem. Chem. Phys., 2015, 17, 2311.

(11) Bässler, H., Köhler, A.; "Hot or cold": how do charge transfer states at the donor-acceptor interface of an organic solar cell dissociate? Phys. Chem. Chem. Phys., 2015, 17, 28451.

(12) Vandewal, K.; Albrecht, S.; Hoke, E. T.; Graham, K. R.; Widmer, J.; Douglas, J. D.; Schubert, M.; Mateker, W. R.; Bloking, J. T.; Burkhard, G. F.; et al. Efficient Charge Generation by Relaxed Charge-Transfer States at Organic Interfaces. Nature Mater. 2013, 13, 63-68.

(13) Etzold, F., Howard, I. A.; Mauer, R.; Meister, M.; Kim, T.-D.; Lee, K.-S.; Baek, N. S.; Laquai, F.; Ultrafast Exciton Dissociation Followed by Nongeminate Charge Recombination in PCDTBT:PCBM Photovoltaic Blends, $J$. Am. Chem. Soc. 2011, 133, 9469-9479.

(14) Graham, K. R.; Ndjawa, G. O. N.; Conron, S. M.; Munir, R.; Vandewal, K.; Chen, J. J.; Sweetnam, S.; Thompson, M. E.; Salleo, A.; McGehee, M. D.; et al. The Roles of Structural Order and Intermolecular Interactions in Determining Ionization Energies and Charge-Transfer State Energies in Organic Semiconductors. Adv. Energy Mater. 2016, 6, 1601211.

(15) Vandewal, K. Interfacial Charge Transfer States in Condensed Phase Systems. Annu. Rev. Phys. Chem. 2016, 67, 113-133.

(16) Graetzel, M.; Janssen, R. A. J.; Mitzi, D. B.; Sargent, E. H. Materials Interface Engineering for Solution-Processed Photovoltaics. Nature 2012, 488, 304-312.

(17) Brédas, J.-L.; Norton, J. E.; Cornil, J.; Coropceanu, V. Molecular Understanding of Organic Solar Cells: The Challenges. Acc. Chem. Res. 2009, 42, 1691-1699.

(18) Deibel, C.; Strobel, T.; Dyakonov, V. Role of the Charge Transfer State in Organic Donor-Acceptor Solar Cells. Adv. Mater. 2010, 22, 4097-4111.

(19) Kang, I.; Yun, H.; Chung, D. S.; Kwon, S.; Kim, Y. Record High Hole Mobility in Polymer Semiconductors via Side-Chain Engineering. J. Am. Chem. Soc. 2013, 135, 1489614899.

(20) Sonar, P.; Singh, S. P.; Li, Y.; Ooi, Z.-E.; Ha, T.; Wong, I.; Soh, M. S.; Dodabalapur, A. High Mobility Organic Thin Film Transistor and Efficient Photovoltaic Devices Using Versatile Donor-acceptor Polymer Semiconductor by Molecular Design. Energy Environ. Sci. 2011, 4, 2288.

(21) Salzmann, I.; Moser, A.; Oehzelt, M.; Breuer, T.; Feng, X.; Juang, Z.; Nabok, D.; Della Valle, R. G.; Duhm, S.; Heimel, G.; et al. Epitaxial Growth of $\pi$-Stacked Perfluoropentacene on Graphene-Coated Quartz. ACS Nano 2012, 6, 10874-10883.

(22) Rinn, A.; Breuer, T.; Wiegand J.; Beck M.; Hübner J.; Döring R. C.; Oestreich M.; Heimbrodt W.; Witte, G.; Chatterjee, S. Interfacial Molecular Packing Determines Exciton Dynamics in Molecular Heterostructures: The Case of Pentacene-Perfluoropentacene. ACS Appl. Mater. Interfaces 2017, 9, 42020-42028.

(23) Hill, H. M.; Rigosi, A. F.; Roquelet, C.; Chernikov, A.; Berkelbach, T. C.; Reichman, D. R.; Hybertsen, M. S.; Brus, L. E.; Heinz, T. F. Observation of Excitonic Rydberg States in
Monolayer MoS2 and WS2 by Photoluminescence Excitation Spectroscopy. Nano Lett. 2015, 15, 2992-2997.

(24) Salzmann, I.; Duhm, S.; Heimel, G.; Oehzelt, M.; Kniprath, R.; Johnson, R. L.; Rabe, J. P.; Koch, N. Tuning the Ionization Energy of Organic Semiconductor Films: The Role of Intramolecular Polar Bonds. J. Am. Chem. Soc. 2008, 130, 12870-12871.

(25) D’Avino, G., Duhm, S., Della Valle, R. G., Heimel, G., Oehzelt, M. Kera, S., Ueno, N., Beljonne, D., Salzmann, I. Electrostatic Interactions Shape Molecular Organization and Electronic Structure of Organic Semiconductor Blends, Chem. Mater. 2020, 32, 1261-1271.

(26) Hinderhofer, A.; Frank, C.; Hosokai, T.; Resta, A.; Gerlach, A.; Schreiber, F. Structure and morphologz of coevaporated pentacene-perfluoropentacene thin films, J. Chem. Phys., 2011, 134, 104702.

(27) Duhm, S., Salzmann, I., Heimel, G., Oehzelt, M., Haase, A., Johnson, R. L., Rabe, J. P., Koch, N., Controlling energy level offsets in organic/organic heterostructures using intramolecular polar bonds. Appl. Phys. Lett. 2009, 94, 033304

(28) Breuer, T.; Witte, G. Thermally Activated Intermixture in Pentacene-Perfluoropentacene Heterostructures. J. Chem. Phys. 2013, 138, 114901.

(29) Becke, A. D.; Density-functional thermochemistry. III. The role of exact exchange, J. Chem. Phys. 1993, 98, 5648-5652.

(30) Becke, A. D.;;A new mixing of Hartree-Fock and local density-functional theories J. Chem. Phys. 1993, 98, 1372-1377.

(31) Klessinger, M; Michl, J, Excited States and Photochemistry of Organic Molecules, John Wiley \& Sons, 1995

(32) Creutz, C.; Newton, M.D.; Sutin, N., Metal-ligand and metal-metal coupling elements J. Photochem. Photo-biol., 1994, 82, 47-59

(33) Kazmaier, P. M.; Hoffmann, R.; A Theoretical Study of Crystallochromy. Quantum Interference Effects in the Spectra of Perylene Pigments, J. Am. Chem. Soc., 1994, 116, 9684-9691

(34) Oehzelt, M., Akaike, K., Koch, N., Heimel, G., Energylevel alignment at organic heterointerfaces, Science Advances 2015, 1, e1501127.

(35) Bouchoms, I. P. M.; Schoonveld, W. A.; Vrijmoeth, J.; Klapwijk, T. M. Morphology Identification of the Thin Film Phases of Vacuum Evaporated Pentacene on SIO2 Substrates. Synth. Met. 1999, 104, 175-178.

(36) Kowarik, S.; Gerlach, A.; Hinderhofer, A.; Milita, S.; Borgatti, F.; Zontone, F.; Suzuki, T.; Biscarini, F.; Schreiber, F. Structure, Morphology, and Growth Dynamics of PerfluoroPentacene Thin Films. Phys. status solidi - Rapid Res. Lett. 2008, 2, 120-122.

(37) Lee, W. H.; Park, J.; Sim, S. H.; Lim, S.; Kim, K. S.; Hong, B. H.; Cho, K. Surface-Directed Molecular Assembly of Pentacene on Monolayer Graphene for High-Performance Organic Transistors. J. Am. Chem. Soc. 2011, 133, 4447-4454.

(38) Götzen, J.; Käfer, D.; Wöll, C.; Witte, G. Growth and Structure of Pentacene Films on Graphite: Weak Adhesion as a Key for Epitaxial Film Growth. Phys. Rev. B 2010, 81, 85440.

(39) Siegrist, T.; Kloc, C.; Schön, J. H.; Batlogg, B.; Haddon, R. C.; Berg, S.; Thomas, G. A. Enhanced Physical Properties in a Pentacene Polymorph. Angew. Chemie Int. Ed. 2001, 40, 1732-1736.

(40) Breuer, T.; Witte, G. Controlling Nanostructures by Templated Templates: Inheriting Molecular Orientation in Binary Heterostructures. ACS Appl. Mater. Interfaces 2015, 7, 20485-20492. 
(41) TURBOMOLE V7.0 2015, a development of University of Karlsruhe and Forschungszentrum Karlsruhe GmbH, 1989-2007, TURBOMOLE GmbH, since 2007; available from http://www.turbomole.com.

(42) Becke, A. D. Density-functional exchange-energy approximation with correct asymptotic behavior, Phys. Rev. A 1988, 38, 3098-3100.

(43) Lee, C., Yang, W., Parr, R. G., Development of the Colle-Salvetti correlation-energy formula into a functional of the electron density. Phys. Rev. B 1988, 37, 785-789.

(44) Becke, A. D., A new mixing of Hartree-Fock and local density-functional theories J. Chem. Phys. 1993, 98, 1372-1377.

(45) Stephens, P. J.; Devlin, F. J.; Chabalowski, C. F.; Frisch, M. J., Ab Initio Calculation of Vibrational Absorption and Circular Dichroism Spectra Using Density Functional Force Fields, J. Phys. Chem. 1994, 98, 11623-11627.

(46) Weigend, F.; Ahlrichs, R. Balanced basis sets of split valence, triple zeta valence and quadruple zeta valence quality for $\mathrm{H}$ to Rn: Design and assessment of accuracy. Phys. Chem. Chem. Phys. 2005, 7, 3297-3305.

(47) Neese F.; The ORCA program system WIREs Comput. Mol. Sci. 2012, 2, 73-78

(48) Yanai T.; Tew D. P.; Handy N. C.; A new hybrid exchange-correlation functional using the Coulombattenuating method (CAM-B3LYP) Chem. Phys. Lett., 2004, 393, 51-57.

(49) McWeeny, R., The density matrix in many-electron quantum mechanics I. Generalized product functions. Factorization and physical interpretation of the density matrices. Proc. R. Soc. Lond. A., 1959, 253, 242-258.

(50) Mehler, E., Self-consistent, nonorthogonal group function approximation for polyatomic systems. I. Closed Shells, J. Chem. Phys., 1977, 67, 2728-2739.

(51) Löwdin, P.-O., Quantum Theory of Many-Particle Systems. I. Physical Interpretations by Means of Density Matrices, Natural Spin-Orbitals, and Convergence Problems in the Method of Configurational Interaction, Phys. Rev., 1955, 97, 1474-1489.

(52) Schäfer, A.; Horn H., Ahlrichs, R. Fully Optimized Contracted Gaussian Basis Sets for Atoms Li to Kr. A, J. Chem. Phys., 1992, 97, 2571. 